PROCEEDINGS OF THE

AMERICAN MATHEMATICAL SOCIETY

Volume 124, Number 10, October 1996

\title{
ON A CONJECTURE OF DANZER AND GRÜNBAUM
}

\author{
MEIR KATCHALSKI AND DAVID NASHTIR
}

(Communicated by Jeffry N. Kahn)

\begin{abstract}
The main result of the paper is that if $A$ is a family of homothetic triangles in the plane such that any 9 of them can be pierced by two points, then all members of $A$ can be pierced by two points. This is best possible in more than one sense: (1) the number 9 cannot be replaced by $8 ;(2)$ no similar statement is true for homothetic copies (or even translates) of a symmetric convex hexagon.
\end{abstract}

\section{InTRODUCTION AND STATEMENT OF RESULTS}

A family of sets is $\Pi^{n}$, or $n$-pierceable, if there exists a set of $n$ points, such that each member of the family contains at least one of the points. The family is $\Pi_{k}^{n}$ if each subfamily of size $k$ or less satisfies $\Pi^{n}$; it is $-\Pi^{n}$ if it is not $\Pi^{n}$.

Helly's theorem asserts that $\Pi_{d+1}^{1}$ implies $\Pi^{1}$ for any family $\mathcal{A}$ of compact convex sets in Euclidean $d$-space $R^{d}$. Consult [1] and [3] for Helly's theorem and its many relatives.

Danzer and Grünbaum studied in [2] intersection properties of boxes with sides parallel to the axis in $R^{d}$. They proved:

D. G. Theorem. If $h=h(d, n)$ is the smallest integer such that $\Pi_{h}^{n}$ implies $\Pi^{n}$ for any family of boxes in $R^{d}$ with sides parallel to the axis, then $h(d, 2)=3 d$ for odd $d$ and $h(d, 2)=3 d-1$ for even $d$.

Here (section 2) a related result on families of homothetic triangles in the plane is proved.

Theorem 1. (i) If $\mathcal{A}$ is any family of homothets of a triangle in $R^{2}$, then $\Pi_{9}^{2}$ implies $\Pi^{2}$ for $\mathcal{A}$.

(ii) There exists in $R^{2}$ a family of 9 translates of a triangle $T$ satisfying $\Pi_{8}^{2}$, but not $\Pi^{2}$.

Note that $S$ is homothetic to $B$ if there exists an $a \in R^{d}$ and an $\alpha>0$ such that $S=a+\alpha B$. (Sometimes such an $S$ is called a positive homothet of $B$.)

In the last section of [2], Danzer and Grünbaum raised two conjectures:

Conjecture 1. If $K$ is any convex polytope in $R^{d}$, then there exists an integer $k=k(K, d)$ such that $\Pi_{k}^{2}$ implies $\Pi^{2}$ for any family of translates of $K$ in $R^{d}$.

Received by the editors July 18, 1994.

1991 Mathematics Subject Classification. Primary 52A35.

The first author's research was supported by the Fund for Promotion of Research at the Technion (grant 100-806) and the Technion V. P. R. Fund (grant 100-934).

(C)1996 American Mathematical Society 
Conjecture 2. If $D$ is a set of $m$ directions in the plane, then $\Pi_{3 m}^{2}$ implies $\Pi^{2}$ for any family $\mathcal{A}$ of convex polygons in the plane, all edges of which have directions belonging to $D$.

In section 3 a counterexample to both conjectures is constructed: For any positive integer $k$ a planar family of $2 k+3$ translates of a centrally symmetric hexagon satisfying $\Pi_{2 k+2}^{2}$ but not $\Pi^{2}$.

The last section describes related results.

\section{Proof of Theorem 1}

The following definitions will be used.

Two halfplanes are related if one of them contains the other. Related halfplanes are ordered by inclusion so that the smaller one is contained in the bigger one.

A convex $n$-gon $B$ is related to the convex $m$-gon $A$ if each of the $n$-halfplanes whose intersection is equal to $B$, is related to one of the $m$-halfplanes whose intersection is equal to $A$. Note that this implies that $n \leq m$. The family $\mathcal{B}$ is related to $A$ if every member of $\mathcal{B}$ is related to $A$.

Note that triangle $B$ is related to triangle $A$ if and only if $B$ is homothetic to $A$.

A set $Q$ pierces a set $A$ if $A \cap Q \neq \varnothing$. The set $Q$ pierces a family of sets $\mathcal{A}$ if it pierces every $A \in \mathcal{A} ; x$ pierces $\mathcal{A}$ if $\{x\}$ pierces $\mathcal{A}$.

By an $m$-gon we mean, unless stated otherwise, a convex $m$-gon.

Let $X^{+}$denote a halfplane whose boundary is the line $X$.

Proof of Theorem 1(i). Let $\mathcal{A}$ be a family of triangles related to a triangle $T$ and satisfying $\Pi_{9}^{2}$. Using compactness arguments, it is sufficient to prove part (i) for $\mathcal{A}$ of finite cardinality.

Let $\mathcal{A}=\left\{A_{i}: i \in[n]\right\},[n]=\{1,2,3, \ldots, n\}$, where $A_{i}=H_{i}^{+} \cap V_{i}^{+} \cap D_{i}^{+}$and $\left\{H_{i}^{+} ; i \in[n]\right\},\left\{D_{i}^{+} ; i \in[n]\right\},\left\{V_{i}^{+} ; i \in[n]\right\}$ are three families of related halfplanes.

For $A=A_{i}$ let $D^{+}(A)=D_{i}^{+}, H^{+}(A)=H_{i}^{+}$, and $V^{+}(A)=V_{i}^{+}$.

For $\mathcal{C} \subseteq \mathcal{A}$ let $D^{+}(\mathcal{C})=\min \left\{D^{+}(A) ; A \in \mathcal{C}\right\}, H^{+}(\mathcal{C})=\min \left\{H^{+}(A) ; A \in \mathcal{C}\right\}$, and $V^{+}(\mathcal{C})=\min \left\{V^{+}(A) ; A \in \mathcal{C}\right\}$.

Let $D^{+}=D^{+}(\mathcal{A}), V^{+}=V^{+}(\mathcal{A})$, and $H^{+}=H^{+}(\mathcal{A})$ with $H^{+}=H^{+}\left(T_{3}\right)$, $V^{+}=V^{+}\left(T_{2}\right)$ and $D^{+}=D^{+}\left(T_{1}\right)$.

Let $\mathcal{T}=\left\{T_{1}, T_{2}, T_{3}\right\}$.

Let $a_{1}=H \cap V, a_{2}=H \cap D$ and $a_{3}=V \cap D$.

Assume that $\mathcal{A}$ is $-\Pi^{1}$, for otherwise $\Pi^{1} \Rightarrow \Pi^{2}$ (see Figure 1).

The proof relies on two simple observations:

Observation 1. For $\mathcal{C} \subseteq \mathcal{A}$ there exists a $\widetilde{\mathcal{C}} \subseteq \mathcal{C}$ with $|\widetilde{\mathcal{C}}| \leq 3$ and $\cap \widetilde{\mathcal{C}}=\bigcap \mathcal{C}$. Furthermore, if $\mathcal{C} \cap \mathcal{T} \neq \varnothing$, then such a $\widetilde{\mathcal{C}}$ can be chosen so that $\widetilde{\mathcal{C}} \cap \mathcal{T} \neq \varnothing$.

Observation 2. If $\{i, j, k\}=\{1,2,3\}$ and $x$ pierces $\mathcal{B}$ with $\left\{T_{i}, T_{j}\right\} \subseteq \mathcal{B}$, then $a_{k}$ pierces $\mathcal{B}$.

Observation 1 holds since $\bigcap \mathcal{C}=D^{+}(\mathcal{C}) \cap H^{+}(\mathcal{C}) \cap V^{+}(\mathcal{C})$ and if $D^{+}(\mathcal{C})=D^{+}(F)$, $H^{+}(\mathcal{C})=H^{+}(G)$, and $V^{+}(G)=V^{+}(K)$, with $\widetilde{\mathcal{C}}=\{F, G, K\} \subseteq \mathcal{C}$, then $\widetilde{\mathcal{C}}$ is as desired.

Observation 2 holds since if w.l.o.g. $x$ pierces $\mathcal{B}$ with $\left\{T_{1}, T_{2}\right\} \subseteq \mathcal{B}$ and $A \in \mathcal{B}$, then $x \in A \cap T_{1} \cap T_{2}$. Then $x \in D^{+} \cap V^{+}$and since $T_{1} \cap T_{2} \subset D^{+} \cap V^{+}$, this implies $a_{3} \in A$, for otherwise $A \cap D^{+} \cap V^{+}=\varnothing$, a contradiction. 


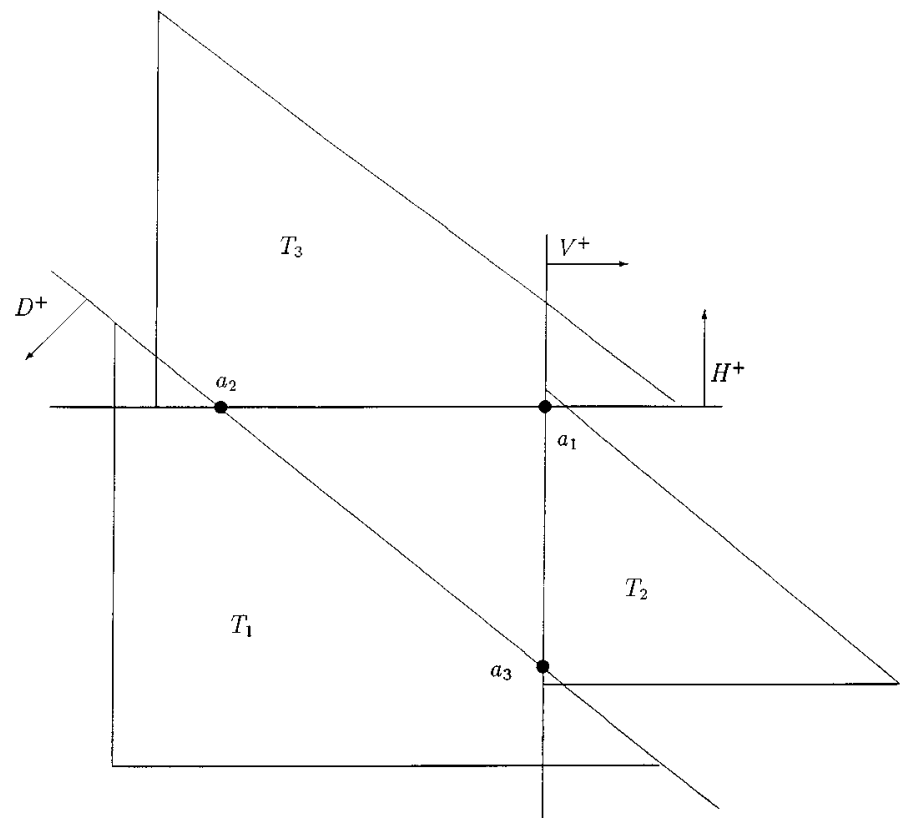

FIGURE 1

Let $\mathcal{A}_{i}=\left\{A \in \mathcal{A}: a_{i} \notin A\right\}$ for $i=1,2,3$. Note that $T_{i} \in \mathcal{A}_{i}$ for $i=1,2,3$. We claim that:

Claim. For $i=1$ or 2 or $3, \mathcal{A}_{i}$ is $\Pi^{1}$.

The claim clearly completes the proof. Otherwise $\bigcap \mathcal{A}_{i}=\varnothing$ for $i=1,2,3$ and by Observation 1 , for $i=1,2,3$ there exists a $\mathcal{C}_{i} \subseteq \mathcal{A}_{i}$, with $\left|\mathcal{C}_{i}\right| \leq 3, \cap \mathcal{C}_{i}=\varnothing$ and $\mathcal{C} \cap \mathcal{T} \neq \varnothing$.

Let

$$
\mathcal{S}=\mathcal{T} \cup \mathcal{C}_{1} \cup \mathcal{C}_{2} \cup \mathcal{C}_{3}
$$

Then $|\mathcal{S}| \leq 3+2+2+2=9$. So $\left(\mathcal{A}\right.$ is $\left.\Pi_{9}^{2}\right), \mathcal{T}$ is $\Pi^{2}$ and a 2 set $\{x, y\}$ pierces $\mathcal{S}$. Assume w.l.o.g. that $x$ pierces $\left\{T_{1}, T_{2}\right\}$. By Observation $2\left\{a_{3}, y\right\}$ pierces $\mathcal{S}$ and $y$ pierces $\mathcal{C}_{3}$, a contradiction, since $\cap \mathcal{C}_{3}=\varnothing$.

Proof of Theorem 1(ii). A family $\mathcal{A}=\left\{A_{i}: 1 \leq i \leq 9\right\}$ of 9 translates of the triangle $T=\{(x, y): x \geq 0, y \geq 0, x+y \leq 7\}$ satisfying $\Pi_{8}^{2}$ but not $\Pi^{2}$ is constructed as follows: for $1 \leq i \leq 9, A_{i}=a_{i}+T$, with

$$
\begin{array}{lll}
a_{1}=(0,-1), & a_{2}=(6,0), & a_{3}=(-1,6), \\
a_{4}=(0,1), & a_{5}=(4,1), & a_{6}=(0,4), \\
a_{7}=(4,0), & a_{8}=(1,0), & a_{9}=(1,4)
\end{array}
$$

(see Figure 2). 

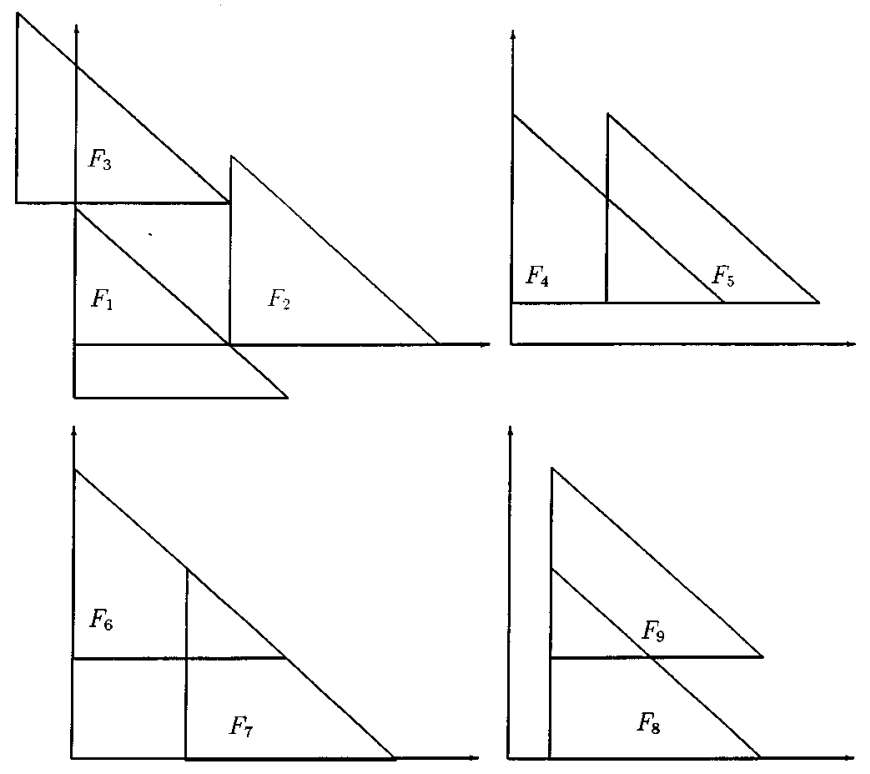

Figure 2

Let $\overline{\mathcal{A}}(i)=\mathcal{A} \backslash\left\{A_{i}\right\}$ for $1 \leq i \leq 9$. Then,

$$
\begin{array}{lll}
\{(6,6),(4,4)\} \in \overline{\mathcal{A}}(1), \quad\{(4,7),(6,0)\} \in \overline{\mathcal{A}}(4), & \{(1,4),(6,6)\} \in \overline{\mathcal{A}}(7), \\
\{(0,6),(4,4)\} \in \overline{\mathcal{A}}(2), \quad\{(1,7),(6,0)\} \in \overline{\mathcal{A}}(5), & \{(7,4),(0,6)\} \in \overline{\mathcal{A}}(8), \\
\{(6,0),(4,4)\} \in \overline{\mathcal{A}}(3), \quad\{(4,1),(6,6)\} \in \overline{\mathcal{A}}(6), & \{(7,1),(0,6)\} \in \overline{\mathcal{A}}(9),
\end{array}
$$

so $\mathcal{A} \in \Pi_{8}^{2}$.

Since $A_{1} \cap A_{2}=\{(6,0)\}, A_{1} \cap A_{3}=\{(0,6)\}$ and $A_{2} \cap A_{3}=\{(6,6)\}$, then a set $\mathcal{B}$ of size 2 that pierces $\mathcal{A}$ contains one of the points $(6,0)$ or $(0,6)$ or $(6,6)$. But $(0,6) \notin A_{2} \cup A_{8} \cup A_{9}$ and $A_{2} \cap A_{8} \cap A_{9}=\varnothing$, so $(0,6) \notin B$. Also $(0,6) \notin$ $A_{3} \cup A_{4} \cup A_{5}$ and $A_{3} \cap A_{4} \cap A_{5}=\varnothing$, so $(6,0) \notin B$. Finally, $(6,6) \notin A_{1} \cup A_{6} \cup A_{7}$ and $A_{1} \cap A_{6} \cap A_{7}=\varnothing$, so $(6,6) \notin B$. So there is no set $B$ of size 2 that pierces $\mathcal{A}$ and $\mathcal{A}$ is not $\Pi^{2}$.

\section{Constructions}

Danzer and Grünbaum's theorem asserts that $\Pi_{5}^{2}$ implies $\Pi^{2}$ for planar families related to a square (the members of such a family are necessarily rectangles with sides parallel to the sides of the square). It is natural to attempt to generalize and prove a similar theorem for planar families of convex sets related to a hexagon. In view of the following construction these attempts cannot succeed.

The construction consists of a family of translates of a centrally symmetric hexagon.

Construction 1 . Consider a $2 \times 2$ square with sides parallel to the axis. Let $c$ and $f$ be opposite vertices with $f$ above and to the right of $c$. Let $a, b, d$ and $e$ be the midpoints of the four sides of the square with $a$ above $d$ and $e$ to the right of $b$.

Let $H$ denote the centrally symmetric hexagon with vertices $a, b, c, d, e$ and $f$. 


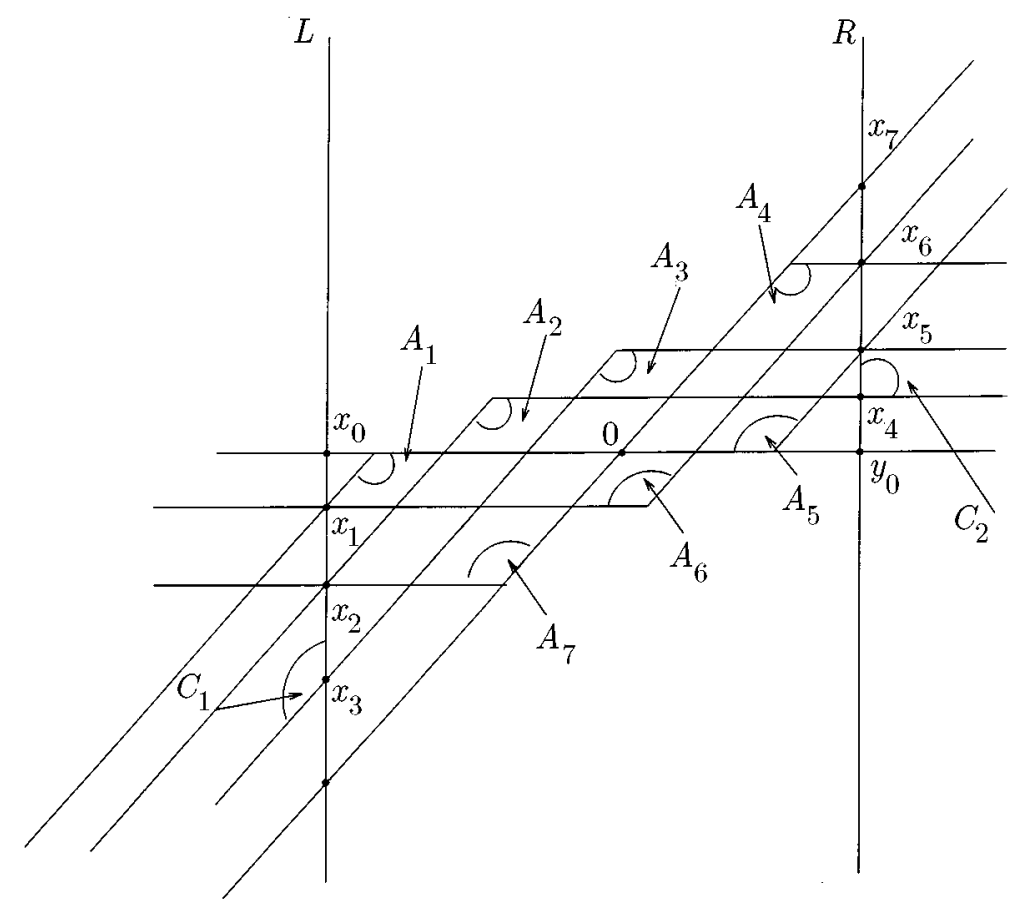

Figure 3

Let $L$ and $R$ be two vertical lines with distance between them equal to 1 and with $L$ to the left of $R$. Let $x_{0}$ and $y_{0}$ lie on a horizontal line with $x_{0}$ on $L$ and $y_{0}$ on $R$.

Let $k$ be a positive integer and let $z_{0}$ on $L$ and $x_{2 k+1}$ on $R$ lie on a line of slope 1 (and therefore parallel to segment $a b$ of the hexagon $H$ ) that contains the midpoint 0 of segment $x_{0} y_{0}$.

Let $x_{1}, \ldots, x_{k}$ lie on $L$ between $x_{0}$ and $z_{0}$ with $x_{i+1}$ below $x_{i}$ for $1 \leq i \leq k-1$.

Let $x_{k+1}, x_{k+2}, \ldots, x_{2 k}$ lie on $R$ between $y_{0}$ and $x_{2 k+1}$ with $x_{k+i+1}$ above $x_{k+i}$ for $1 \leq i \leq k$ (see Figure 3 where $k=3$ ). Define translates $A_{1}, \ldots, A_{2 k+1}, C_{1}$ and $\mathrm{C}_{2}$ of $\mathrm{H}$ as follows:

The $a b$-side (corresponding to side $a b$ of $H$ ) of $A_{i}$ contains $x_{i}$ for $1 \leq i \leq k$.

The $a b$-side of $A_{k+1}$ contains 0 .

The $a f$-side of $A_{1}$ contains 0 .

The $a f$-side of $A_{i}$ contains $x_{i+k-1}$ for $2 \leq i \leq k+1$.

The $e d$-side of $A_{i}$ contains $x_{i}$ for $k+2 \leq i \leq 2 k+1$.

The $c d$-side of $A_{k+i+1}$ contains $x_{i-1}$ for $1 \leq i \leq k$.

Finally:

The $e$-vertex of $C_{1}$ is $x_{k}$.

The $c$-vertex of $C_{2}$ is $x_{k+1}$ (see Figure 3 ).

However, in the figure, suitable angles of the hexagons and not the complete hexagons are sketched (the $a$-angle of $A_{i}$ for $1 \leq i \leq k+1$, the $d$-angle of $A_{i}$ for $k+2 \leq i \leq 2 k+1$, the $c$-angle of $C_{2}$ and the $e$-angle of $C_{1}$ ).

Let $\mathcal{A}=\left\{A_{1}, A_{2}, \ldots, A_{2 k+1}, C_{1}, C_{2}\right\}$. It remains to show that

Theorem 2. The family $\mathcal{A}$ of Construction 2 is $\Pi_{2 k+2}^{2}$ but not $\Pi^{2}$. 
Proof. For $X \in \mathcal{A}$ let $\overline{\mathcal{A}}(X)$ denote $\mathcal{A} \backslash\{X\}$. All the $A_{i}$ 's contain 0 so $\left\{0, x_{k+1}\right\}$ pierces $\overline{\mathcal{A}}\left(C_{1}\right)$ and $\left\{0, x_{k}\right\}$ pierces $\overline{\mathcal{A}}\left(C_{2}\right)$. Since $A_{i}$ contains $\left\{x_{i}, x_{i+1}, \ldots, x_{i+k-1}\right\}$ (with addition modulo $2 k+1$ ), it follows that $\left\{x_{i+k}, x_{i+2 k}\right\}$ pierces $\mathcal{A} \backslash\left\{A_{i}, C_{1}, C_{2}\right\}$. But $\left\{x_{i+k}, x_{i+2 k}\right\}$ pierces $\overline{\mathcal{A}}\left(A_{i}\right)$ for $2 \leq i \leq 2 k+1$. However, $\left\{x_{0}, x_{k+1}\right\}$ pierces $\overline{\mathcal{A}}\left(A_{1}\right)$. So

$$
\mathcal{A} \text { is } \Pi_{2 k+2}^{2} \text {. }
$$

Assume that $\mathcal{A}$ is $\pi^{2}$ and that $\{u, v\}$ pierces $\mathcal{A}$. Since $C_{1}$ and $C_{2}$ are disjoint, assume that $u \in C_{1}$ and $v \in C_{2}$. It is easy to check that if we project $u$ and $v$ horizontally to $L$ and $R$ respectively, then the set consisting of the two projections pierces $\mathcal{A}$. It is also easy to check that a point of $L \cap C_{1}$ as well as a point of $R \cap C_{2}$ pierces at most $k$ members of $\left\{A_{1}, \ldots, A_{2 k+1}\right\}$. Consequently, $\{u, v\}$ pierces at most $2 k+2$ members of $\mathcal{A}$, a contradiction, implying that $\mathcal{A}$ is not $\Pi^{2}$.

\section{Related Results}

Related results may be found in [4], the M.Sc. Thesis of David Nashtir (written under the supervision of Meir Katchalski).

\section{(1) A generalization of Theorem 1.}

Theorem. Let $P$ be a convex pentagon with two pairs of parallel sides. If $\mathcal{A}$ is any planar family of convex sets related to $P$ and if $\mathcal{A}$ is $\Pi_{9}^{2}$, then $\mathcal{A}$ is $\Pi^{2}$.

The proof is very technical.

\section{(2) Constructions.}

Construction 2. A convex quadrilateral $Q$ and for every positive integer $k$ a family $\mathcal{A}$ of $2 k+3$ translates of $Q$ satisfying $\Pi_{2 k+2}^{2}$ but not $\Pi^{2}$.

The construction is similar to Construction 1 .

Construction 3 . For every positive integer $k$ a planar family of $3 n+1$ triangles related to a triangle $T$ and satisfying $\Pi_{3 n}^{3}$ but not $\Pi^{3}$.

Additional results will appear in Nashtir's Ph.D. thesis [5] (written under the supervision of Katchalski). It is proved there that there is no positive integer $k$ such that $\Pi_{k}^{2}$ implies $\Pi^{2}$ for every family of translates of a fixed nondegenerate simplex in $R^{3}$.

Other implications of $\Pi_{k}^{2}$ for families of convex sets are also studied there.

\section{REFERENCES}

1. L. Danzer, B. Grünbaum and V. Klee, Helly's theorem and its relatives, Proc. Symposia in Pure Math., vol. VII (Convexity) (1963), 101-180. MR 28:524

2. L. Danzer and B. Grünbaum, Intersection properties of boxes in $R^{d}$, Combinatorica 2 (1982), 237-246. MR 84g:52014

3. J. Eckhoff, Helly Radon and Carathéodory Type Theorems, Chapter 2.1 in P. M. Gruber and J. M. Wills (eds.), Handbook of Convex Geometry, North-Holland (1993), 389-448. MR 94k:52010

4. D. Nashtir, On a conjecture of Danzer and Grünbaum, Ms.C. Thesis, Technion, Haifa (1990), (Hebrew)

5. _ Helly type problems, Ph.D. Thesis, in preparation.

Department of Mathematics, Technion-Israel Institute of Technology, Haifa 32000 , ISRAEL

E-mail address: meirk@tx.technion.ac.il 